\title{
Mudas de tamarindos irrigadas com diferentes tipos e proporções de água
}

\author{
Production of seedlings tamarinds irrigated with different types of water \\ Plántulas de tamarindos de regadío con diferentes tipos y proporciones de agua
}

Recebido: 05/08/2021 | Revisado: 10/08/2021 | Aceito: 13/08/2021 | Publicado: 16/08/2021

Ranieri Antunes de Queiroga

ORCID: https://orcid.org/0000-0001-9862-5073

Instituto Federal de Educação Ciência e Tecnologia da Paraíba, Brasil

E-mail: raniery.queiroga@ifpb.edu.br

Ednaldo Barbosa Pereira Junior

ORCID: https://orcid.org/0000-0003-0098-3206

Instituto Federal de Educação Ciência e Tecnologia da Paraíba, Brasil

E-mail: ebprj2@hotmail.com

Joserlan Nonato Moreira

ORCID: https://orcid.org/0000-0002-2290-7119

Instituto Federal de Educação Ciência e Tecnologia da Paraíba, Brasil

E-mail: joserlan.moreira@ifpb.edu.br

Ranniery Felix dos Santos

ORCID: https://orcid.org/0000-0003-3146-2112 Instituto Federal de Educação Ciência e Tecnologia da Paraíba, Brasil

E-mail: rannierylevita@hotmail.com

Pedro Lima Filho

ORCID: https://orcid.org/ 0000-0001-7009-1735 Instituto Federal de Educação Ciência e Tecnologia da Paraíba, Brasil

E-mail: pedro.lima@ifpb.edu.br

Francisco de Sales Oliveira Filho

ORCID: https://orcid.org/0000-0002-4276-862X

Instituto Federal de Educação, Ciência e Tecnologia da Paraíba, Brasil

E-mail: francisco.filho@ifpb.edu.br

Diego Ernani Leite Bezerra

ORCID: https://orcid.org/0000-0002-0896-9516 Instituto Federal de Educação, Ciência e Tecnologia da Paraíba, Brasil E-mail: diego.bezerra@ifpb.edu.br

João Ferreira Neto

ORCID: https://orcid.org/0000-0001-5327-3799 Instituto Federal de Educação, Ciência e Tecnologia da Paraíba, Brasil E-mail: joao.ferreira@ifpb.edu.br

José de Sousa Brito Filho

ORCID: https://orcid.org/0000-0001-5343-1056 Instituto Federal de Educação, Ciência e Tecnologia da Paraíba, Brasil E-mail: jose.brito@ifpb.edu.br

\begin{abstract}
Resumo
As precipitações pluviométricas irregulares e a elevada evapotranspiração são características da região semiárida do Nordeste Brasileiro. O conjunto dessas variáveis fazem da água um fator limitante para agricultura irrigada, o que torna a reutilização necessária, principalmente em locais sob condições de crescente escassez. Este trabalho teve o objetivo de avaliar o desenvolvimento de mudas de tamarindos e sua influência nos atributos químicos do solo, em função dos diferentes tipos e proporções de águas. A pesquisa foi desenvolvida no setor de produção de mudas localizado no Instituto Federal da Paraíba, Campus Sousa. Utilizou-se o delineamento de blocos inteiramente casualizados, com cinco tratamentos e quatro repetições. Os tratamentos resultaram das seguintes combinações: $\mathrm{T} 1=$ $100 \%$ Poço Artesiano (PA), T2 =100\% Ar-Condicionado (AC), T3 = Combinação 50\% Ar Condicionado + 50\% Poço Artesiano (AC+PA), T4 = Combinação $50 \%$ Efluente Agroindustrial $+50 \%$ Ar Condicionado $(\mathrm{EA}+\mathrm{AC})$ e T5 = $100 \%$ Efluente Agroindustrial (EA). As mudas de tamarindo foram produzidas em sacos plásticos, utilizando Neossolo Flúvico e esterco na proporção (2:1) como substrato. Foram avaliadas as seguintes variáveis: altura de planta, diâmetro de caule, número de folhas, massa verde e seca da parte aérea e raiz, comprimento da raiz e influência nos atributos do solo (pH, P, K, Na e MO). A combinação de 50\% das águas de ar condicionado e 50\% efluente agroindustrial se mostrou adequada na produção de mudas de Tamarindo. O efluente agroindustrial é recomendável para irrigação de plântulas de tamarindo, apesar dos elevados teores de sódio e cloreto. O uso da água de ar condicionado mostrou-se viável na produção de mudas de tamarindos.
\end{abstract}

Palavras-chave: Reutilização; Solo; Efluente; Ar-condicionado. 


\begin{abstract}
Irregular pluvimetric precipitations and high evapotranspiration are characteristic of the semiarid region of northeastern Brazil. The set of these variables make water a limiting factor for irrigated agriculture, which makes reuse necessary, especially in places under conditions of growing scarcity. This work aimed to propose the use of water from air conditioning and agroindustrial effluent in the production and development of Tamarind seedlings. The research was developed in the seedling production sector located at the Federal Institute of Paraíba, Campus Sousa. A completely randomized block design was used, with five treatments and four replications. The treatments resulted from the following combinations: $\mathrm{T} 1=100 \%$ Artesian well $(\mathrm{PA}), \mathrm{T} 2=100 \%$ air conditioning $(\mathrm{AC}), \mathrm{T} 3=$ combination 50\% air conditioning $+50 \%$ Artesian well $(\mathrm{AC}+\mathrm{PA}), \mathrm{T} 4=$ combination 50\% Agroindustrial effluent + $50 \%$ air conditioning $(\mathrm{EA}+\mathrm{AC})$ and $\mathrm{T} 5=100 \%$ Agroindustrial Effluent $(\mathrm{EA})$. Tamarind seedlings were produced in plastic bags, using Neossol Fluvic and manure in proportion (2:1) as substrate. The following variables were evaluated: plant height, stem diameter, number of leaves, green and dry mass of the aerial part and root, root length and influence on soil attributes ( $\mathrm{pH}, \mathrm{P}, \mathrm{K}, \mathrm{Na}$ and $\mathrm{OM}$ ). The combination of $50 \%$ air conditioning water and $50 \%$ agro-industrial effluent proved to be adequate for the production of Tamarindo seedlings. The agro-industrial effluent is recommended for irrigation of tamarind seedlings, despite the high levels of sodium and chloride. The use of air conditioning water proved to be viable in the production of tamarind seedlings.
\end{abstract}

Keywords: Reuse; Soil; Effluent; Air Conditioning.

\title{
Resumen
}

Las lluvias irregulares y la alta evapotranspiración son características de la región semiárida del noreste de Brasil. El conjunto de estas variables hacen del agua un factor limitante para la agricultura de regadío, lo que hace necesaria su reutilización, especialmente en lugares en condiciones de creciente escasez. Este trabajo tuvo como objetivo evaluar el desarrollo de plántulas de tamarindo y su influencia en los atributos químicos del suelo, en función de diferentes tipos y proporciones de agua. La investigación se realizó en el sector de producción de plántulas ubicado en el Instituto Federal de Paraíba, Campus Sousa. Se utilizó un diseño de bloques completamente al azar, con cinco tratamientos y cuatro repeticiones. Los tratamientos resultaron de las siguientes combinaciones: T1 = 100\% Pozo Artesiano (PA), T2 $=100 \%$ Aire Acondicionado $(\mathrm{AC}), \mathrm{T} 3=$ Combinación 50\% Aire Acondicionado + 50\% Pozo Artesiano (AC + PA), $\mathrm{T} 4=$ Combinación $50 \%$ Efluente Agroindustrial + 50\% Aire Acondicionado (EA + AC) y T5 = 100\% Efluente Agroindustrial (EA). Las plántulas de tamarindo se produjeron en bolsas plásticas, utilizando Fluvic Neosol y estiércol (2: 1) como sustrato. Se evaluaron las siguientes variables: altura de la planta, diámetro del tallo, número de hojas, masa verde y seca de brotes y raíces, longitud de la raíz e influencia en los atributos del suelo (pH, P, K, Na y MO). La combinación de 50\% de agua de aire acondicionado y 50\% de efluente agroindustrial resultó ser adecuada para la producción de plántulas de Tamarindo. El efluente agroindustrial se recomienda para el riego de plántulas de tamarindo, a pesar de los altos niveles de sodio y cloruro. El uso de agua de aire acondicionado demostró ser viable en la producción de plántulas de tamarindo.

Palabras clave: Reutilización; Suelo; Efluente; Aire acondicionado.

\section{Introdução}

No Brasil a fruticultura é um dos setores de maior destaque no agronegócio, o país é um dos três maiores produtores mundiais de frutas. Em 2016, o valor da produção de frutíferas chegou a R \$ 33,3 bilhões, o maior da série histórica, iniciada em 1974. Em relação a 2015, o valor da produção de frutíferas teve um acréscimo de 26,0\%, sua maior alta desde 2001. Os seis principais produtos concentram $73,2 \%$ do valor da produção nacional: laranja $(25,1 \%)$, banana $(25,0 \%)$, abacaxi $(7,3 \%)$, uva $(6,4 \%)$, maçã $(5,0 \%)$ e mamão $(4,4 \%)$ (IBGE, 2016).

A fruticultura é um setor agrícola de suma importância para o Brasil, representando excelente atrativo na diversificação de atividades, e exercendo ao mesmo tempo papel econômico, social e alimentar. As frutas desempenham papel importante, pois são fontes de proteínas, carboidratos, vitaminas, sais minerais, água e celulose, o que leva uma maior consciência das populações, sobre a importância do consumo de alimentos saudáveis, na prevenção de doenças e na melhoria da qualidade de vida, razão pela qual o consumo de frutas vem aumentando dia a dia (Aragão et al.,2002)

O tamarindeiro (Tamarindus indica L.) pertence à família das fabáceas, subfamília Caesalpinoideae, sendo originário da África Tropical, de onde se dispersou. É encontrado em várias regiões brasileiras, adaptado aos diversos estados, e plantado dispersadamente, sendo considerado fruto típico (Sousa et al. 2010). Os indivíduos que crescem nos trópicos derivam de 
sementes coletadas, ao acaso, na África e na Índia, as quais são, portanto, destituídas de melhoramento genético. Ainda assim, o tamarindeiro desponta como cultura atrativa e de grande futuro comercial (Seagri, 2010).

Os recursos hídricos têm importância fundamental no desenvolvimento de diversas atividades econômicas. Em relação à produção agrícola, a água pode representar até 90\% da constituição física das plantas (Sales, 2014). Um dos grandes desafios dos recursos hídricos é equilibrar a demanda com a disponibilidade da água existente, para isso uma solução alternativa é o reaproveitamento de águas residuárias, principalmente na agricultura. Pois na maioria das áreas agrícolas irrigadas o volume de água utilizado é superior ao realmente necessário para a produção (Faggion, Oliveira; Christofidis, 2009)

Avaliando ecofisiologia de mudas de pinheira (Annonasquamosa L.) sob doses de esterco bovino e lâminas de irrigação, Silva et al. (2020) constatou que a utilização de esterco bovino e o manejo da irrigação influenciou positivamente as trocas gasosas da pinheira em sua fase de mudas, em condições de casa de vegetação.

Por ser o tamarindeiro considerado moderadamente tolerante à salinidade no estágio de muda, a cultura pode ser inserida como espécie potencialmente viável para avaliar a influência do manejo na redução dos efeitos negativos da salinidade durante o crescimento das plantas em áreas semiáridas (Gebauer et al., 2004; Gurumurthy et al., 2007).

De acordo com Ayres e Westcot (1991) a redução da disponibilidade de recursos hídricos de boa qualidade e o aumento da produção de despejos domésticos e industriais tem impulsionado o uso de águas de qualidade inferior na irrigação, cuja avaliação se torna necessária.

Kummer et al. (2012) afirmam que a utilização de efluentes na agricultura cresceu consideravelmente nos últimos anos em muitos países, inclusive no Brasil; no entanto, ainda não foram suficientemente estudados todos os aspectos positivos e negativos dessa técnica, especialmente sobre as propriedades físicas e químicas do solo, absorção de nutrientes pelas plantas ou sua toxidez.

Tem-se buscado métodos alternativos de reutilização da água, como o aproveitamento das águas pluviais, água-cinza, águas residuais tratadas e a dessalinização, que aparecem como meios de conservação da água e como alternativas para enfrentar a falta desse recurso, tanto para fins potáveis quanto não potáveis, tornando uma opção prática e a baixo custo para minimizar a escassez (Pushard, 2008).

Desta forma, o objetivo da pesquisa foi de avaliar o desenvolvimento de mudas de tamarindo e sua influência nos atributos químicos do solo em função de diferentes tipos de águas e suas proporções.

\section{Metodologia}

O experimento foi conduzido no viveiro de mudas do Instituto Federal de Educação Ciência e Tecnologia da Paraíba, Campus - Sousa-PB, Unidade São Gonçalo, com 220 metros de altitude, de coordenadas geográfica latitude 645’33" Sul e longitude $38^{\circ} 13^{\prime} 41^{\prime}$. Segundo os registros do Banco de Dados Meteorológicos para Ensino e Pesquisa (BDMEP - INMET, 2019) para o período citado, não ocorreram precipitações, as temperaturas médias de mínima e máxima de $21,04^{\circ} \mathrm{C}$ e $33,86^{\circ} \mathrm{C}$, respectivamente e a umidade relativa do ar média foi de aproximadamente $64,45 \%$.

$\mathrm{O}$ delineamento experimental utilizado foi de blocos inteiramente casualizados, com cinco tratamentos e quatro repetições. A parcela experimental foi composta por três plantas. Os tratamentos resultaram em T1 $=100 \%$ Poço Artesiano (PA), $\mathbf{T} 2=100 \%$ Ar Condicionado (AC), T3 = Combinação 50\% Ar Condicionado $+50 \%$ Poço Artesiano (AC+PA), $\mathbf{T} 4=$ Combinação $50 \%$ Efluente Agroindustrial + 50 \% Ar Condicionado (EA+AC) e T5 = 100 \% Efluente Agroindustrial (EA).

As coletas das águas proveniente dos sistemas de ar condicionado foi feita de acordo com (Pereira Junior et al. 2017), o efluente Agroindustrial (Ferreira Neto, et al., 2017), foi com base de trabalhos desenvolvidos por esses autores, que serviram de instrumento para essa pesquisa e a do poço localizado próximo ao Bloco de Agroindústria. 
Foram realizadas três coletadas de amostras das águas utilizadas para a irrigação (dos tratamentos) para realizar análise química, a primeira no início do tratamento, segunda com 45 dias e a terceira com 90 dias após a semeadura (DAS). As análises foram feitas pelo Laboratório de Análises de Solo, Água e Planta do IFPB, Campus Sousa, cujos resultados encontrase na (Tabela 1).

Na produção das mudas foram usadas como materiais propagativos sementes selecionadas de tamarindo (Indica $L$.) de exemplares dentro do próprio Instituto Federal da paraíba, Campus Sousa. O substrato usado foi proveniente da combinação de um neossolo flúvico e esterco na proporção (2:1 v), condicionados em recipientes (sacos plásticos de 2 1) preenchidos manualmente e conduzidos ao viveiro. Em seguida, foi coletada uma amostra do substrato para análise química feita pelo Laboratório de Análises de Solo, Água e Planta, do IFPB Campus Sousa - PB, cujo resultado encontra-se na (Tabela 2).

Tabela 1: Análise química das águas utilizadas para irrigação das mudas de tamarindos (dados médios das três coletas), IFPB, Campus Sousa - PB 2019.

\begin{tabular}{|c|c|c|c|c|c|c|c|c|c|c|c|c|c|c|}
\hline Fonte & $\mathbf{p H}$ & $\begin{array}{c}\mathrm{CE} \\
\mathrm{dsm}^{-1}\end{array}$ & $\mathbf{K}$ & $\mathbf{N a}$ & Ca & Mg & $\begin{array}{c}\mathrm{SO}_{4} \\
-\cdots\end{array}$ & $\begin{array}{l}\mathrm{CO}_{3}{ }^{2} \\
-(\mathrm{mm}\end{array}$ & $\begin{array}{c}\mathrm{HCO} \\
3 \\
\mathrm{c})^{0,5}=\end{array}$ & $\begin{array}{l}\mathrm{CL}^{-} \\
- \\
--\end{array}$ & CSR & $\mathrm{NaCl}$ & $\mathrm{CaCO}_{3}$ & $\begin{array}{c}\text { RAS } \\
(\text { mmolc })\end{array}$ \\
\hline PA & 8,0 & 0,98 & 1,33 & 10,1 & 2,3 & 2,0 & 0,37 & 0,50 & 7,0 & 4,4 & 6,0 & 467 & 388 & 6,11 \\
\hline $\mathrm{AC}$ & 6,9 & 0,05 & 0,22 & 0,06 & 0,16 & 0,1 & 0,09 & 0,00 & 3,8 & 0,7 & 3,6 & 16,6 & 18,8 & 0,09 \\
\hline $\begin{array}{l}\mathrm{AC}+ \\
\mathrm{PA}\end{array}$ & 8,0 & 0,54 & 0,83 & 3,4 & 1,5 & 1,2 & 0,14 & 0,42 & 6,2 & 2,2 & 3,6 & 265 & 226 & 2,94 \\
\hline $\begin{array}{l}\mathrm{EA}+ \\
\mathrm{AC}\end{array}$ & 5,9 & 0,65 & 1,3 & 4,3 & 0,9 & 0,6 & 0,28 & 0,00 & 3,6 & 5,4 & 5,3 & 311 & 263 & 4,72 \\
\hline EA & 5,5 & 1,23 & 3,7 & 11,2 & 2,2 & 1,0 & 0,41 & 0,00 & 6,4 & 5,8 & 4,9 & 576 & 487 & 8,4 \\
\hline
\end{tabular}

$\mathrm{PA}=$ Poço Artesiano $; \mathrm{AC}=\mathrm{Ar}$ condicionado $\mathrm{AC}+\mathrm{PA}=50 \% \mathrm{Ar}$ condicionado $+50 \%$ Poço Artesiano $; \mathrm{EA}+\mathrm{AC}=50 \%$ Efluente agroindustrial $+50 \%$ Ar condicionado; EA= Efluente agroindustrial. Fonte: Autores.

Tabela 2: Análise química do substrato utilizado na produção de mudas de tamarindos, IFPB, Campus Sousa - PB 2019.

\begin{tabular}{|c|c|c|c|c|c|c|c|c|c|c|c|}
\hline pH & $\mathbf{P}$ & $\mathbf{K}$ & $\mathbf{N a}$ & $\mathbf{C a}$ & Mg & Al & $\mathrm{H}+\mathrm{Al}$ & SB & CTC & MO & PST \\
\hline $\mathrm{H}_{2} \mathrm{O}$ & $\mathrm{mg} \mathrm{dm}^{-3}$ & &.-- & 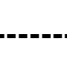 & --.-. & $\mathrm{Cmol}^{\mathbf{c}} \mathbf{d m}^{-3}$ & --- & ----.. & & $\mathrm{g} \mathrm{kg}^{-1}$ & $\%$ \\
\hline 7,4 & 882 & 3,7 & 0,7 & 7,4 & 3,3 & 0,00 & 0,00 & 15,1 & 15,1 & 37,7 & 5 \\
\hline
\end{tabular}

Fonte: Autores.

$\mathrm{Na}$ semeadura foram colocadas três sementes por recipiente, na profundidade de $2 \mathrm{~cm}$, cobrindo-as com fina camada de substrato e casca de arroz para conservar a umidade e favorecer a germinação, com posterior raleamento, deixando-se a plântula mais vigorosa e cortando as demais rentes ao substrato com auxílio de uma tesoura.

Durante a condução do experimento foram feitas irrigações diárias (manhã), fornecendo um volume de água suficiente para elevar a umidade do substrato próximo à capacidade de campo. As plantas espontâneas foram eliminadas manualmente, assim que as mesmas surgiram. Durante o período de condução do experimento, não foi feita nenhuma adubação em cobertura.

Aos 30, 60 e 90 (DAS) foram avaliadas as características morfológicas: altura de plantas - AP (cm) (obtido pela distância entre a região do colo e a gema apical do ramo principal), diâmetro do caule (mm) - DC (obtido pela medição das plantas a quatro centímetros acima do colo, com o auxílio de um paquímetro digital) e número de folhas - NF (obtido pela contagem total do número de folhas totalmente expandidas).

Na última avaliação aos 90 (DAS) foram determinadas em g. planta ${ }^{-1}$, a massa fresca da parte aérea (MFPA), massa fresca da raiz (MFR), massa seca da parte aérea (MSPA) e da raiz (MSR) (obtida pela separação da raiz através de um corte na 
região do colo, acondicionadas em sacos de papel, etiquetadas e secadas em estufa a $65^{\circ} \mathrm{C}$, com circulação forçada de ar até peso constante) e comprimento da raiz, com auxilio de uma régua.

Concluindo do experimento foram coletados amostras compostas do solo, em cada parcela experimental para determinar o pH e os teores de Fósforo $\left(\mathrm{P} \mathrm{mg} \mathrm{dm}^{-3}\right)$, Potássio $\left(\mathrm{K} \mathrm{cmol}_{\mathrm{c}} \mathrm{dm}^{-3}\right)$, Sódio $\left(\mathrm{Na}_{\mathrm{cmol}} \mathrm{dm}^{-3}\right)$ e matéria orgânica $(\mathrm{MO} \mathrm{g}$ $\mathrm{kg}^{-1}$ ) do solo com o intuito de verificar as mudanças ocorridos durante o período experimental, analisada conforme a metodologia (EMBRAPA 1997) no laboratório de Solo, Água e Planta do IFPB, Campus Sousa.

Os resultados foram submetidos à Análise de Variância (ANOVA) e as médias, comparadas pelo teste de Tukey, a 0,05 de probabilidade, através do programa computacional - SISVAR (Ferreira, 2014).

\section{Resultados e Discussão}

Para altura de plantas (Tabela 3), a maior diferença significativa está entre os tratamentos T1 e T4, 5,74 cm (aos 30 DAS), 12,9 cm (aos 60 DAS) e 16,3 cm (aos 90 DAS). A menor altura aos 90 (DAS) foi registrada no T1 com 34,27 cm e a maior no T4 com 50,57 cm, que difere de Nobrega et al. (2017) com mudas de goiabeira irrigadas com diferentes tipos de água, onde o tratamento com água de ar condicionado superou o efluente agroindustrial na variável altura de planta.

Lima Neto et. al. (2015) observou que o biofertilizante comum favoreceu o crescimento de mudas de tamarindos até a condutividade elétrica máxima estimada da água de $1,1 \mathrm{dS} \mathrm{m}^{-1}$ referente a maior altura de $58,9 \mathrm{~cm}$, enquanto, a irrigação com água de salinidade superior a $1,1 \mathrm{dS} \mathrm{m}^{-1}$ prejudicou o crescimento das mudas referidas. Comparativamente, neste experimento tratamento T5 com Condutividade elétrica (CE) 1,23 dS m${ }^{-1}$ (Tabela 2) não interferiu de forma drástica, pelo contrário favoreceu o crescimento das mudas de tamarindos.

Geralmente a redução na altura da planta ocorre devido à água salina reduzir o potencial osmótico do solo, e consequentemente a energia livre da água, diminuindo a absorção de água pela planta e a turgescência das células, a qual afeta as taxas de elongação e divisão celular, que reflete diretamente no crescimento das plantas ocasionando esta redução (Ashraf; Harris, 2004).

Tabela 3: Médias de altura de mudas de tamarindo, irrigadas com diferentes tipos e proporções de água, IFPB, Campus Sousa, 2019.

\begin{tabular}{cccc}
\hline & \multicolumn{3}{c}{ ALTURA DE PLANTA (cm) } \\
Tratamentos & 30 & 60 & 90 \\
T1 & $7,30 \mathrm{c}$ & $12,85 \mathrm{c}$ & $34,27 \mathrm{c}$ \\
T2 & $8,55 \mathrm{ab}$ & $18,62 \mathrm{~b}$ & $42,12 \mathrm{~b}$ \\
T3 & $9,45 \mathrm{ab}$ & $18,37 \mathrm{~b}$ & $39,95 \mathrm{~b}$ \\
T4 & $12,77 \mathrm{a}$ & $25,75 \mathrm{a}$ & $50,57 \mathrm{a}$ \\
T5 & $11,55 \mathrm{ab}$ & $25,07 \mathrm{a}$ & $45,47 \mathrm{~b}$ \\
\hline F & $*$ & $*$ & $*$ \\
\hline CV \% & 9,18 & 8,8 & 6,83 \\
\hline
\end{tabular}

$\mathrm{T} 1=$ Poço Artesiano; $\mathrm{T} 2=$ Ar condicionado; $\mathrm{T} 3=50 \% \mathrm{Ar}$ condicionado $+50 \%$ Poço Artesiano; $\mathrm{T} 4=50 \%$ Efluente agroindustrial + $50 \% \mathrm{Ar}$ condicionado; T5= Efluente agroindustrial. Médias seguidas, em linha, com letras diferentes apresentaram efeitos significativo a nível de $(\mathrm{p}<0,050)$ pelo teste Tukey. Fonte: Autores. 
O T4 demonstrou melhor comportamento para variável diâmetro de caule aos 30 (DAS), já os Tratamentos T2 e T3 apresentaram os menores resultados no mesmo período, enquanto aos 60 e 90 (DAS) os tratamentos apresentaram comportamento semelhante para essa variável conforme (Tabela 4),

Avaliando Produção de mudas de essências florestais irrigadas com água de abastecimento, rejeito salino e efluente da piscicultura, Sousa (2014) não verificou diferença significativa para o diâmetro do colmo para angico, sabiá, tamarindo, jucá e mulungú.

Em seu trabalho analisando fontes e níveis de salinidade em mudas de mamoeiro Cavalcante et al. (2010) detectaram que o crescimento em altura e o diâmetro do caule das plantas foram prejudicados com o aumento da condutividade elétrica da água de irrigação.

Tabela 4: Médias de diâmetro do caule de mudas de tamarindo, irrigadas com diferentes tipos e proporções de água, IFPB, Campus Sousa, 2019.

\begin{tabular}{cccc}
\hline & \multicolumn{3}{c}{ DIÂMETRO DO CAULE (mm) } \\
Tratamentos & 30 & 60 & 90 \\
T1 & $1,70 \mathrm{ab}$ & $2,16 \mathrm{a}$ & $3,69 \mathrm{a}$ \\
T2 & $1,51 \mathrm{c}$ & $2,39 \mathrm{a}$ & $4,22 \mathrm{a}$ \\
T3 & $1,42 \mathrm{c}$ & $2,50 \mathrm{a}$ & $3,90 \mathrm{a}$ \\
T4 & $1,80 \mathrm{a}$ & $2,91 \mathrm{a}$ & $4,70 \mathrm{a}$ \\
T5 & $1,56 \mathrm{bc}$ & $2,63 \mathrm{a}$ & $4,32 \mathrm{a}$ \\
\hline $\mathrm{F}$ & $*$ & Ns & Ns \\
\hline CV \% & 5,11 & 13,30 & 12,64 \\
\hline
\end{tabular}

$\mathrm{T} 1=$ Poço Artesiano; $\mathrm{T} 2=$ Ar condicionado $; \mathrm{T} 3=50 \%$ Ar condicionado $+50 \%$ Poço Artesiano $; \mathrm{T} 4=50 \%$ Efluente agroindustrial $+50 \%$ Ar condicionado; T5= Efluente agroindustrial. Médias seguidas, em linha, com letras diferentes apresentaram efeitos significativo a nível de $(\mathrm{p}<0,050)$ pelo teste Tukey. Fonte: Autores.

Aos 30, 60 e 90 (DAS) para variável número de folhas (NF) o Tratamento T1 apresentou os menores resultados, os melhores resultados nestes mesmos períodos foram apresentados pelo tratamento T4, os Tratamento T4 e T5 apesar de apresenta o maiores CEa bem acima dos demais tratamentos (Tabela 1), refletiu numa maior quantidade de folhas comparado com demais tratamentos.

Trabalho com comportamento contrário foram verificados por Pinheiro et al. (2017) que observaram que houve efeito significativo dos níveis de salinidade da água de irrigação sobre o número de folhas , o aumento da salinidade da água de irrigação afetou positivamente o NF de mudas de mamoeiro, as plantas que foram submetidas a irrigação com CEa de 3,5 dS $\mathrm{m}^{-1}$ apresentaram acréscimos de 6,94\% em comparação com o menor nível salino (CEa $0,3 \mathrm{dS} \mathrm{m}^{-1}$ ), como as de condutividade elétrica igual ou maior que 3,0 dS m m $^{-1}$ também oferecem restrição severa ao crescimento inicial do tamarindeiro (Cavalcante et al 2012). 
Tabela 5: Médias de Número de Folhas (NF) de mudas de tamarindo, irrigadas com diferentes tipos e proporção de água, IFPB, Campus Sousa, 2019.

\begin{tabular}{cccc}
\hline & \multicolumn{3}{c}{ NÚMERO DE FOLHAS } \\
Tratamentos & ---10 & 60 & 90 \\
T1 & 30 & $17 \mathrm{c}$ & $36 \mathrm{c}$ \\
T2 & $9 \mathrm{c}$ & $20 \mathrm{~b}$ & $48 \mathrm{~b}$ \\
T3 & $12 \mathrm{~b}$ & $21 \mathrm{~b}$ & $49 \mathrm{~b}$ \\
T4 & $12 \mathrm{~b}$ & $26 \mathrm{a}$ & $61 \mathrm{a}$ \\
T5 & $15 \mathrm{a}$ & $24 \mathrm{a}$ & $53 \mathrm{a}$ \\
\hline F & $14 \mathrm{ab}$ & $*$ & $*$ \\
\hline CV $\%$ & $*$ & 4,70 & 19,91 \\
\hline
\end{tabular}

$\mathrm{T} 1=$ Poço Artesiano; $\mathrm{T} 2=\mathrm{Ar}$ condicionado; $\mathrm{T} 3=50 \%$ Ar condicionado $+50 \%$ Poço Artesiano; $\mathrm{T} 4=50 \%$ Efluente agroindustrial + $50 \% \mathrm{Ar}$ condicionado; T5= Efluente agroindustrial. Médias seguidas, em linha, com letras diferentes apresentaram efeitos significativo a nível de $(\mathrm{p}<0,050)$ pelo teste Tukey. Fonte: Autores.

Com relação ao comprimento da raiz (CR) o tratamento que obteve o melhor resultado foi o Tratamento T4, os demais tratamentos não diferiram estatisticamente entre si, conforme os resultados expostos na (Tabela 6).

Avaliando Produção de mudas de essências florestais irrigadas com água de abastecimento, rejeito salino e efluente da piscicultura, Sousa (2014) não verificou diferença significativa para o comprimento da raiz para angico, sabiá, tamarindo, jucá e mulungú.

Os resultados da massa fresca da parte aérea (MFPA), massa fresca da raiz (MFR), massa seca parte aérea (MSPA) e massa seca da raiz (MSR) não diferiram estatisticamente entre os tratamentos, conforme os resultados exposto na (Tabela 6). Verifica-se que os tratamentos de maior teor salino (T1 e T5) não influenciaram, tanto de forma positiva e nem negativa nos seus dados comparando com os demais. Provavelmente em razão da pequena duração experimental ao ponto de não expressar resultados diferentes nessas variáveis. Testando os mesmos tratamentos e variáveis Pereira Junior et al (2018) constatou comportamento semelhante no desenvolvimento inicial de mudas de cajueiro.

As folhas são órgãos sensíveis que, em meios adversamente salinos, sofrem redução em tamanho e número (Gebauer et al., 2004; Medeiros et al., 2011), resultando em menor área foliar (Hardikar; Pandey, 2011) e, com efeito, em perdas da atividade clorofilática e fotossintéticas como verificado por Oliva et al. (2008) e Hunsche et al. (2010) em mudas de tamarindeiro. 
Tabela 6: Médias de comprimento radicular, massa fresca da parte aérea, massa fresca da raiz, massa seca da parte aérea e massa seca da raiz de mudas de tamarindos, aos 90 DAS, irrigados com diferentes tipos e proporções de água, IFPB, Campus Sousa, 2019.

\begin{tabular}{cccccc}
\hline \multirow{2}{*}{ Tratamentos } & $\begin{array}{c}\text { Comprimento da } \\
\text { raiz }\end{array}$ & Aérea & Raiz & Massa Seca & Raiz \\
\hline T1 & $39,04 \mathrm{~b}$ & $0,81 \mathrm{a}$ & $0,21 \mathrm{a}$ & $0,28 \mathrm{a}$ & $0,12 \mathrm{a}$ \\
$\mathrm{T} 2$ & $36,83 \mathrm{~b}$ & $0,90 \mathrm{a}$ & $0,20 \mathrm{a}$ & $0,31 \mathrm{a}$ & $0,12 \mathrm{a}$ \\
$\mathrm{T} 3$ & $36,83 \mathrm{~b}$ & $0,87 \mathrm{a}$ & $0,20 \mathrm{a}$ & $0,30 \mathrm{a}$ & $0,12 \mathrm{a}$ \\
$\mathrm{T} 4$ & $47,08 \mathrm{a}$ & $0,92 \mathrm{a}$ & $0,20 \mathrm{a}$ & $0,31 \mathrm{a}$ & $0,12 \mathrm{a}$ \\
$\mathrm{T} 5$ & $38,08 \mathrm{~b}$ & $0,84 \mathrm{a}$ & $0,21 \mathrm{a}$ & $0,28 \mathrm{a}$ & $0,13 \mathrm{a}$ \\
\hline $\mathrm{F}$ & $*$ & $\mathrm{~ns}$ & $\mathrm{Ns}$ & $\mathrm{Ns}$ & $\mathrm{Ns}$ \\
\hline $\mathrm{CV} \%$ & 10,14 & 7,85 & 10,06 & 7,84 & 5,84 \\
\hline
\end{tabular}

$\mathrm{T} 1=$ Poço Artesiano; $\mathrm{T} 2=\mathrm{Ar}$ condicionado; $\mathrm{T} 3=50 \%$ Ar condicionado $+50 \%$ Poço Artesiano; $\mathrm{T} 4=50 \%$ Efluente agroindustrial + $50 \% \mathrm{Ar}$ condicionado; T5= Efluente agroindustrial. Médias seguidas, em linha, com letras diferentes apresentaram efeitos significativo a nível de $(\mathrm{p}<0,050)$ pelo teste Tukey. Fonte: Autores.

Houve diferença significativa $(\mathrm{p}<0,05)$ para Potássio $(\mathrm{K})$, Sódio $(\mathrm{Na})$, fosforo $(\mathrm{P})$ e matéria orgânica $(\mathrm{MO})$, exceto para $\mathrm{pH}$ do solo, (Tabela 7).

$\mathrm{O}$ pH do solo, apesar de não apresentar significância entre os tratamentos permaneceram levemente alcalinos, evidenciando que os tratamentos testados nestas condições, são considerados adequados. Segundo (Ayers \& Westcot, 1991) um pH entre 6,5 e 8,4; é adequado, ao equilíbrio nutricional das culturas irrigadas (Tabela 7).

Observa-se na (Tabela 7) que os teores de fósforo (P) não diferiram entre se nos tratamentos (1, 2, 3 e 4), apenas o tratamento 5 diferenciou dos demais, apresentaram teores muito alta, segundo (Alvarez V. et al. 1999). Avaliando os mesmos tratamentos na produção de mudas de mamoeiro, Bezerra et al. (2019), não constatou alterações significativas para os teores de P no solo.

Enquanto que, Sousa et al. (2006) avaliando o reuso de água residuária na produção de pimentão e nas Características químicas do solo não constatou alterações nos teores de fosforo, no solo após o término do experimento localizada no município de Lagoa Seca, PB.

Nota-se na (Tabela 7) que a variação ocorrida entre os tratamentos para os teores de potássio $\left(\mathrm{K}^{+}\right)$no solo, apresentando diferença significativa entre os tratamentos. Com o maior valor expressado pelo T3. Entretanto, era de esperar que o teor de potássio fosse mais elevado irrigados pelos os T4 e T5, devido conterem maiores concentrações desse nutriente (Tabela 1).

Diferentemente encontrado nesse trabalho, Duarte et al. (2008) Verificando o efeito do reuso de efluente tratado no solo concluíram que não provocou alterações significativas no $\mathrm{pH}$, nem nos teores de fósforo e potássio do solo.

Ferreira Neto et al. (2013) avaliando alterações química no solo e produção de beterraba irrigada com água residuária constataram baixa concentração de potássio na água residuária utilizada no experimento, o que contribuiu, em parte, para não influenciar no aumento de potássio no solo.

Em relação ao sódio (tabela 7), os valores mais elevados foram constatados nos tratamentos T1, T4 e T5, o menor teor foi no T2 irrigado com água ar condicionado, esse comportamento do solo é justificável pelas análises feitas nas águas utilizadas nos tratamentos, vindo a interferir na composição desses elementos solo (Tabela 1). Irrigando mudas de pinheira com efluente agroindustrial, Andrade et al. (2020) constatou acréscimo na concentração de Na no solo, material coletado do setor de produção de leite e derivados do Instituto Federal da Paraíba, Campus Sousa. 
Segundo (Herpin et al., 2007; Leal et al., 2009) também constataram aumento nos teores de Na no solo quando utilizaram efluente tratado de origem doméstica, atribuiu esse acúmulo pelo fato de o teor desse elemento no efluente ser elevado.

Tabela 7: Características químicas do solo após o término do experimento, irrigados com diferentes tipos e proporções de águas, IFPB - Sousa-PB, 2019.

\begin{tabular}{lcccccc}
\hline \multicolumn{1}{c}{$\begin{array}{c}\text { Características químicas } \\
\text { (fertilidade do solo) }\end{array}$} & T1 & T2 & T3 & T4 & T5 & CV(\%) \\
\cline { 2 - 7 } & $7,62 \mathrm{a}$ & $7,47 \mathrm{a}$ & $7,57 \mathrm{a}$ & $7,55 \mathrm{a}$ & $7,55 \mathrm{a}$ & 1,00 \\
$\mathrm{pH}$ & $896 \mathrm{~b}$ & $837 \mathrm{~b}$ & $910 \mathrm{~b}$ & $866 \mathrm{~b}$ & $949 \mathrm{a}$ & 3,73 \\
Fósforo $\left(\mathrm{mg} \mathrm{dm}^{-3}\right)$ & $1,09 \mathrm{~b}$ & $1,09 \mathrm{~b}$ & $1,31 \mathrm{a}$ & $1,06 \mathrm{~b}$ & $1,12 \mathrm{~b}$ & 3,02 \\
Potássio $\left(\mathrm{cmol}_{\left.\mathrm{c} \mathrm{dm}^{-3}\right)}\right.$ & $0,65 \mathrm{~b}$ & $0,13 \mathrm{c}$ & $0,40 \mathrm{~b}$ & $1,16 \mathrm{a}$ & $1,50 \mathrm{a}$ & 10,36 \\
Sódio $\left(\mathrm{cmol}_{\mathrm{c}} \mathrm{dm}^{-3}\right)$ & $19,07 \mathrm{~b}$ & $22,87 \mathrm{a}$ & $17,44 \mathrm{~b}$ & $19,01 \mathrm{~b}$ & $19,51 \mathrm{~b}$ & 7,16 \\
Material Orgânico $\left(\mathrm{g} \mathrm{kg}^{-1}\right)$ & &
\end{tabular}

$\mathrm{T} 1=$ Poço Artesiano; $\mathrm{T} 2=\mathrm{Ar}$ condicionado $\mathrm{T} 3=50 \%$ Ar condicionado $+50 \%$ Poço Artesiano; $\mathrm{T} 4=50 \%$ Efluente agroindustrial + 50\% Ar condicionado; T5= Efluente agroindustrial. Médias seguidas, em linha, com letras diferentes apresentaram efeitos significativo a nível de $(\mathrm{p}<0,050)$ pelo teste Tukey. Fonte: Autores.

Segundo os dados dispostos na (Tabela 7), observa-se que o conteúdo de matéria orgânica encontrada no T2, foi superior aos demais tratamentos, permanecendo assim na concentração média que varia de 16 a $30\left(\mathrm{~g} \mathrm{~kg}^{-1}\right)$.

Bezerra et al. (2019), avaliando diferentes tipos de água e efluente agroindustrial em diferentes proporções constatou que o conteúdo de matéria orgânica existente no solo irrigado não apresentou diferença significativa entre os diferentes tratamentos, permanecendo com concentrações muita alta

A matéria orgânica do solo (MOS) é fonte de energia para os microrganismos, aumenta a capacidade de armazenar umidade, reter e fixar fósforo e nitrogênio, aumentar a capacidade de troca de cátions (CTC), ajudar a segurar potássio, cálcio e magnésio, entre outros nutrientes disponíveis para as raízes da planta (PRIMAVESI, 2002).

\section{Conclusão}

A combinação de 50\% das águas de ar condicionado e 50\% efluente agroindustrial se mostrou adequada na produção de mudas de Tamarindo.

O efluente agroindustrial é recomendável para irrigação de plântulas de tamarindo, apesar de elevar os teores de sódio no solo.

O uso da água de ar condicionado mostrou-se viável na produção de mudas de tamarindos.

Como forma de estigar o reuso de diferentes tipos de águas, sugeri o desenvolvimento de outras pesquisas com diferentes frutíferas, como forma de estimular a sustentabilidade e aproveitamento de resíduos.

\section{Referências}

Andrade, E. de, Sarmento, M. I. de A., Pereira Junior, E. B., Moreira, J. N., Nóbrega, Éverson P. da, Caiana, C. R. A., Bezerra Neto, F. das C., Medeiros, A. C. de, \& Maracajá, P. B. (2020) Reutilização de água para produção de mudas de pinha. Ressarce, Society and Development, 9 , e536974403.

Arruda, F. P. et al. (2002) Efeito do estresse hídrico na emissão/abscisão de estruturas reprodutivas do algodoeiro herbáceo cv. CNPA 7H. Revista Brasileira de Engenharia Agrícola e Ambiental, 6, 21-27.

Ashraf, M., \& Harris, P. J. C. (2004) Potential biochemical indicators of salinity tolerance in plants. Plant Science, $166,3-16$.

Alvarez, V., V. H., Novais, R. F., Barros, N. F., Cantarutti, R. B. \& Lopes, A. S. (1999). Interpretação dos resultados das análises de solo. In: Ribeiro, A.C., Guimarães, P. T. G. \& Alvarez V., V. H., eds. Recomendação para o uso de corretivos e fertilizantes em Minas Gerais. 5ª aproximação. CFSEMG, 25-32p. 
Ayers, R. S., Westcoot, D. W. (1991) A qualidade de água na agricultura. UFPB, 218p.

Batista, R. O. et al. (2014) O efeito da água residuária da suinocultura no desenvolvimento e qualidade de mudas de Eucalyptus urophylla. Ciência Florestal, $24,127-135$.

Bezerra D., Filho P. L., Pereira Jr, E. B., Azevedo P. R. E \& Silva, E. A. (2019). Mudas de mamoeiro (Carica Papaya L.) irrigadas com diferentes tipos de água. revista verde de agroecologia e desenvolvimento sustentável. 14(1).

BDMEP - INMET. (2019). Banco de Dados Meteorológicos para Ensino e Pesquisa http://www.inmet.gov.br/portal/index.php?r =estacoes/estacoesAutomaticas.

Cavalcante, L. F., Cordeiro, J. C., Nascimento, J. A. M., Cavalcante, I. H. L., Dias, T. A. (2010) .Fontes e níveis da salinidade da água na formação de mudas de mamoeiro cv. sunrise solo. Semina: Ciências Agrárias, 31, 1281-1290.

CONAMA - Conselho Nacional Do Meio Ambiente - Resolução No. 54, de 28 de novembro de 2005 estabelece critérios gerais para reuso de água potável.

Costa, F. G. B. et al. (2014). Desenvolvimento inicial de cultivares de melão fertirrigadas com distintas proporções de esgoto doméstico em Mossoró-RN. Revista Caatinga, 27, 116-123.

DNOCS - Departamento Nacional De Obras Contra As Secas. (2018). Perímetro irrigado de São Gonçalo. http://www.dnocs.gov.br/ dnocs/doc/cana is/perimetros_irrigados/pb/sao_gon alo.htm

Duarte, A. S. R., Airold, I. P. S., Folegatti, M. V., Botrel, T. A., Soares, T. M. (2008). Efeitos da aplicação de efluente tratado no solo: pH, matéria orgânica, fósforo e potássio. R. Bras. Eng. Agríc. Ambiental, 12, 302-310.

EMBRAPA, Empresa Brasileira de Pesquisa Agropecuária. (1997). Manual de métodos de análise de solo, Centro Nacional de Pesquisa de Solos, $2^{\mathrm{a}}$ ed. Rev. Atual.

Ferreira Neto, J., De Queirós M. M. F., Nobre, R. G., Pereira Junior, E. B., Sousa, J. C., Sousa, J. X. (2017). Caracterização físico-química e microbiológica da beterraba irrigada com efluente agroindustrial. Rev. de Agroecologia no Semiárido. 1, 13 - 23.

Ferreira, D. F. (2014). Sisvar: A Guide for Its Bootstrap Procedures in Multiple Comparisons. Ciência e Agrotecnologia, 38, 109-112.

Fonseca, A. F. (2010). Disponibilidade de nitrogênio, alterações nas características químicas do solo e do milho pela aplicação de efluente de esgoto tratado. 2001. 126f. Dissertação (Mestrado em Agronomia) - Escola Superior de Agricultura "Luiz de Queiroz".

Herpin, U., Gloaguen, T. V., Fonseca, A. F., Montes, C. R., Mendonça, F. C., Piveli, R. P., Breulmann, G., Forti, M. C. \& Melfi, A. J. (2007). Chemical effects on the soil-plant system in a secondary treated wastewater irrigated coffee plantation - A pilot field study in Brazil. Agric. Water Manag., 89:105-115.

Kummer, A. C. B., Silva, I. P. F., Lobo, T. F., Grassi Filho, H. Qualidade da água residuária para irrigação do trigo. In: IV Workshop Internacional De Inovações Tecnológicas Na Irrigação E I Inovagri International Meeting, Fortaleza, Anais... Fortaleza, 2012.

Leal, R. M. P., Herpin, U., Fonseca, A. F., Firme, L. P., Montes, C. R. \& Melfi, A. J. (2009). Sodicity and salinity in a Brazilian Oxisol cultivated with sugarcane irrigated with wastewater. Agric. Water Manag, 96: 307-316.

Malavolta, E., Vitti, G. C., \& Oliveira, S. A. (1989). Avaliação do estado nutricional das plantas: Princípios e aplicações. Piracicaba: POTAFOS, 1989. 201 p.

Malavolta, E., Vitti, G.C., \& Oliveira, S. A. (1997). Avaliação do estado nutricional das plantas: princípios e aplicações. Potafos, Piracicaba, Brasil. 1997, $281 \mathrm{p}$.

Marinho, C. S., Monnerat, P. H., Almy, J R., Carvalho, C., Marins, S. L. D., \& Vieira, A. (2002). Análise química do pecíolo e limbo foliar como indicadora do estado nutricional dos mamoeiros 'solo' e' 'formosa. Scientia Agricola, 59, 373-381.

Mota, T. R., Oliveira, D. M., \& Inada, P. (2012). Utilização da água do sistema de ar condicionado visando o desenvolvimento sustentável. $10^{\circ}$ Forum de Extensãoe cultura da UEM.

Neto, A. J. DE L., Cavalcante, L, F., Nunes, J. C., Souto, A. G. L., \& Bezerra, F. T. C. (2015). Mudas de tamarindeiro irrigadas com água salina em solo sem e com biofertilizantes. Irriga, 20, 730-744.

Nóbrega, E. P., Sarmento, M. I. A., Rodrigues, M. L. M., Oliveira, P. R. R., Neto, J. F., \& Maracajá, P. (2018). Desenvolvimento inicial de mudas de goiabeira irrigadas com diferentes tipos de água. Revista de agroecolgia no Semiárido, 1, 01-09.

Patel, A. D., Panchal, N. S., Pandey, I. B., \& Pandey, A. N. (2010). Growth, water status and nutrien accumulation of seedlings of jatroph curcas in response to soil salinity. Anales de Biología, 32, 59-71.

Pereira Junior, E. B., Sousa, J. F., Lima, C. J., Hafle, O. M., Gomes, D. J., \& Oliveira, F. T. (2017). Quantidade e qualidade da água dos aparelhos condicionadores de ar no IFPB Campus Sousa, PB. Revista de Agroecologia no Semiárido, 1, 1-12.

Pereira Junior, E. P. B., Sousa, P. S., Casimiro, D. E., Filho, F. S. O., \& Lima, F. V. S. (2018). Desenvolvimento inicial de mudas de cajueiro irrigadas com diferentes tipos de água. Brazilian Applied Science Review, 2, 644-656.

Pérez-Alfocea, F., Estañcia, M. T., Santa Cruz, A., \& Bolarin, M. C. (1993). Effets of salinity on nitrate, total nitrogean, soluble protein and freeamino acid levels in tomato plants. Journal Horticulture Science. 68, 1021 - 1027.

Pinheiro, F. W. A., Nobre, R. G., Souza, L. P., Oliveira, S. G., Araújo, J. E. S., \& Veloso, L. L. S. A. (2017). Irrigação com águas salinas e adubação nitrogenada na produção de mudas de mamoeiro "Formosa". Revista Verde de Agroecologia e Desenvolvimento Sustentável, 12, 17-22. 
Research, Society and Development, v. 10, n. 10, e481101019098, 2021

(CC BY 4.0) | ISSN 2525-3409 | DOI: http://dx.doi.org/10.33448/rsd-v10i10.19098

Primavesi, A. (2002). Agricultura em regiões tropicais. Manejo ecológico do solo. Nobel. 549p.

Pushard, T. S. (2008). The State of Rainwater Harvesting In The U.S. On Tap, 20-22.

SEBRAE - Serviço Brasileiro De Apoio Às Micro E Pequenas Empresas - Boletim de Inteligência. Agronegócio Fruticultura, 2015. http://www.sebraemercados.com.br/wp-content/uploads/2015/11/Panorama-do-mercado-de-fruticultura-no-Brasil.pdf.

Silva, D. J. P., (2011). Resíduos da Indústria de Laticínios. Trabalho de Conclusão de Curso (Graduação em Gestão Ambiental) - Departamento de Tecnologia de Alimentos, Universidade Federal Viçosa, Viçosa-MG

Silva, F. de A. da., Almeida Neto, I. P. de., Fernandes, P. D., Dias, M. dos S., Brito, M. E. B., \& Lima, A. M. de. (2020). Ecofisiologia de mudas de pinheira (Annona squamosa L.) sob doses de esterco bovino e lâminas de irrigação. Research, Society and Development, 9, e305974175.

Sousa, J. T., Ceballos, B. S. O., Henrique, I. N., Dantas, J. P., \& Lima, S. M. (2006). Reuso de água residuária na produção de pimentão (Capsicum annuum L. R. Bras. Eng. Agríc. Ambiental, 10, 89-96.

Souza, A. C. M. (2014). Manejo integrado do rejeito da dessalinização da água salobra na agricultura. Dissertação de Mestrado, Programa de PósGraduação em Manejo do Solo e Água. Universidade Federal Rural do Semiárido. 59p.

Souza, J. A. A. et. al. (2011). Contaminação microbiológica do perfil do solo com esgoto sanitário. Acta Scientiarum.Technology, 33, 5-8.

Tedesco, M. J., Gianello, C., Bissani, C. A., Hohnen, H., \& Volkweiss, S. J. (1995). Análise de solo, plantas e outros materiais. Departamento de solos Faculdade de Agronomia Universidade Federal do Rio Grande do Sul, 174p.

Taiz, L., \& Zeiger, E. (2013). Fisiologia vegetal. (5a ed.), ArtMed, 954 p. 